\title{
ARTICLE
}

\section{Size-induced enhanced magnetoelectric effect and multiferroicity in chromium oxide nanoclusters}

D. Halley ${ }^{1}$, N. Najjari ${ }^{1}$, H. Majjad ${ }^{1}$, L. Joly ${ }^{1}$, P. Ohresser² ${ }^{2}$ F. Scheurer ${ }^{1}$, C. Ulhaq-Bouillet ${ }^{1}$, S. Berciaud ${ }^{1}$, B. Doudin ${ }^{1} \&$ Y. Henry ${ }^{1}$

The control of the magnetization of a material with an electric field would make the design and the integration of novel electronic devices possible. This explains the renewed interest in multiferroic materials. Progress in this field is currently hampered by the scarcity of the materials available and the smallness of the magnetoelectric effects. Here we present a proofof-principle experiment showing that engineering large strains through nanoscale size reduction is an efficient route for increasing magnetoelectric coefficients by orders of magnitude. The archetype magnetoelectric material, $\mathrm{Cr}_{2} \mathrm{O}_{3}$, in the form of epitaxial clusters, exhibits an unprecedented $600 \%$ change in magnetization magnitude under $1 \mathrm{~V}$. Furthermore, a multiferroic phase, with both magnetic and electric spontaneous polarizations, is found in the clusters, while absent in the bulk.

\footnotetext{
${ }^{1}$ Institut de Physique et Chimie des Matériaux de Strasbourg, Université de Strasbourg, CNRS UMR 7504, 23 rue du Loess, BP 43, F-67034 Strasbourg Cedex 2, France. ${ }^{2}$ Synchrotron SOLEIL, L'Orme des Merisiers, Saint-Aubin, BP 48, F-91192 Gif-sur-Yvette, France. Correspondence and requests for materials should be addressed to D.H. (email: david.halley@ipcms.unistra.fr).
} 
$\mathrm{V}$ oltage control of spintronic devices is envisioned through the coexisting permanent magnetic and electric dipole moments in multiferroic magnetoelectric materials ${ }^{1,2}$. Related multifunctional devices would greatly benefit from enlarged magnetoelectric coupling, which allows controlling the magnetic properties of devices by applying a bias voltage. Although the magnetoelectric concept dates back to Pierre Curie $^{3}$, in 1894, the number of studied magnetoelectric materials, and a fortiori of magnetoelectric multiferroic materials, is still limited ${ }^{4}$. Furthermore, producing a substantial modification of the magnetization magnitude under electric field is an ongoing challenge. For instance, the archetype magnetoelectric material, $\mathrm{Cr}_{2} \mathrm{O}_{3}$, which is antiferromagnetic and paraelectric, has a magnetoelectric susceptibility of only a few $\mathrm{ps} \mathrm{m}^{-1}$ (ref. 5). Even under the largest electric fields created across dielectric thin films $\left(10^{9} \mathrm{Vm}^{-1}\right)$, the change in magnetization would not exceed a few $10^{-3} \mu_{\mathrm{B}}$ per $\mathrm{Cr}$ atom, that is, about $1 \mathrm{emu} \mathrm{cm}^{-3}$.

Strain engineering is envisioned as a possible route for improving magnetoelectric properties ${ }^{2}$ with the predicted huge increase of the magnetoelectric coefficients ${ }^{6}$ and even the appearance of new multiferroic phases for strained materials ${ }^{6,7}$. Small epitaxial structures, which can sustain much larger amounts of stress than the bulk, are thus worth exploring. Here, we investigate highly strained nanometre size chromium oxide particles, imprisoned in a single crystalline dielectric matrix. We provide evidence for the occurrence of spontaneous magnetization and electric polarization in these particles, which not only behave as superparamagnets but also as superparaelectrics. Moreover, we show that their magnetoelectric coefficients are several orders of magnitude larger than the linear terms in bulk $\mathrm{Cr}_{2} \mathrm{O}_{3}$. Their magnetization can be modified by a fraction of $\mu_{\mathrm{B}}$ per atom, opening the possibility of efficient use of electric field for controlling the magnetization of the material.

\section{Results}

$\mathrm{Fe} / \mathrm{MgO}: \mathrm{Cr} / \mathrm{Fe}$ magnetic tunnel junctions. Insight into the magnetoelectric properties of the nanoparticles is obtained through their tunnel magnetoresistance (TMR) signature (Fig. 1a-c). For this work, indeed, we fabricated modified $\mathrm{Fe} / \mathrm{MgO} / \mathrm{Fe}(001)$ magnetic tunnel junction (MTJ) stacks ${ }^{9}$ by interrupting the growth of the 5- or 5.5-nm thick $\mathrm{MgO}$ insulating layer after $2.5 \mathrm{~nm}$ to allow the deposition of a small amount of chromium. The epitaxial growth of chromium on a smooth $\mathrm{MgO}(001)$ surface follows the Volmer-Weber mode ${ }^{10}$, which results in the formation of nanometric flat clusters, with narrow size distribution (Fig. 2). Following indications of partial oxidation of chromium at the interface with $\mathrm{MgO}$ (ref. 11), we annealed the stack after growth completion (Methods) in order to achieve full oxidation of the clusters embedded in $\mathrm{MgO}$. For a nominal chromium thickness $t_{\mathrm{Cr}}=0.5 \mathrm{~nm}$, the resulting particles are typically $2 \mathrm{~nm}$ thick and $6 \mathrm{~nm}$ wide (Fig. 1d,e).

Their chemical, structural and magnetic properties were studied by X-ray absorption spectroscopy (XAS) and X-ray magnetic circular dichroism (XMCD). Absorption measurements at the $L_{2,3}$ edges of $\mathrm{Cr}$ (Fig. 3a) show no significant contribution of residual metallic chromium. They indicate that around $90 \%$ of $\mathrm{Cr}$ atoms are in $\mathrm{a}+3$ oxidation state and reside in an oxygen octahedral environment alike in bulk $\alpha-\mathrm{Cr}_{2} \mathrm{O}_{3}$. Moreover, the largest part (80\%) of the XMCD signal (Fig. 3b) can be reproduced using the reference spectrum of $\mathrm{Cr}^{3+}$ ions in ruby $\alpha-\mathrm{Al}_{2} \mathrm{O}_{3}: \mathrm{Cr}^{3+}$ (ref. 12), which has the same corundum structure as $\alpha-\mathrm{Cr}_{2} \mathrm{O}_{3}$. Since bulk $\mathrm{Cr}_{2} \mathrm{O}_{3}$ is an antiferromagnet, the sizable $\mathrm{XMCD}$ signal recorded, proving the existence of a finite magnetization $M$, should primarily result from spin canting in a

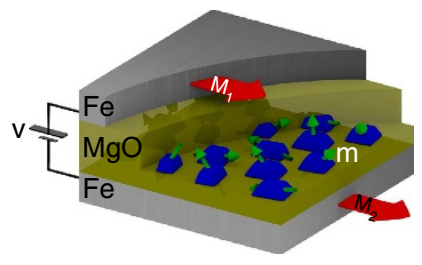

b

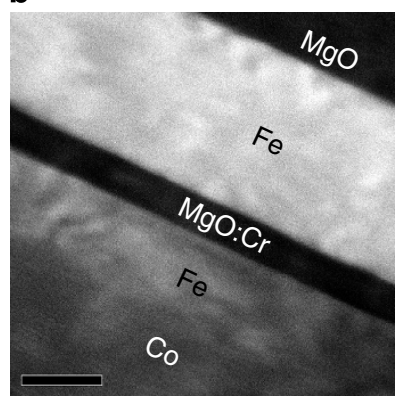

C

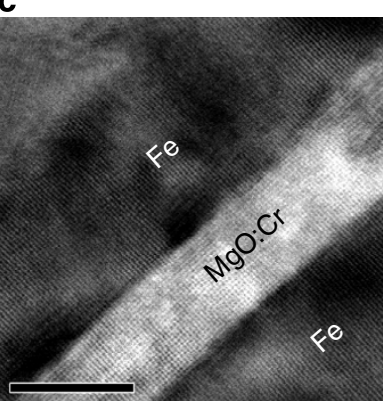

d

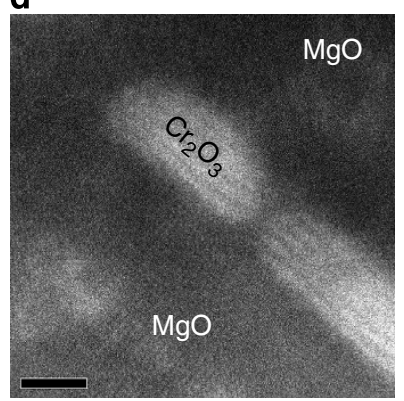

e

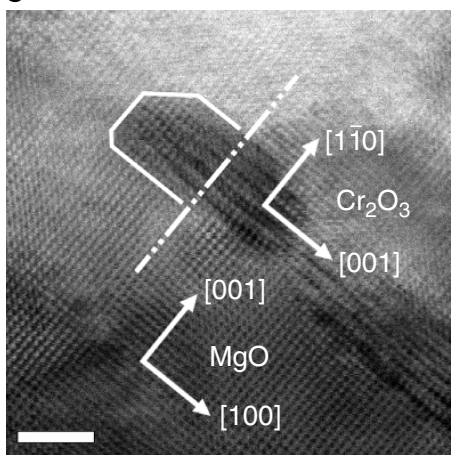

Figure 1 | Chromium oxide clusters embedded in a MgO tunnel barrier. (a) Schematic of the $\mathrm{Fe} / \mathrm{MgO}: \mathrm{Cr} / \mathrm{Fe}$ magnetic tunnel junction devices. $\boldsymbol{M}_{\mathbf{1}}$ and $\boldsymbol{M}_{\mathbf{2}}$ denote the magnetization vectors of the ferromagnetic electrodes, and $\boldsymbol{m}$ that of the superparamagnetic granules inside the tunnel barrier. (b,c) Cross-sectional scanning TEM micrographs of a MTJ stack with a nominal $\mathrm{Cr}$ thickness of $0.4 \mathrm{~nm}$ showing that the inclusion of $\mathrm{Cr}$ in the $\mathrm{MgO}$ barrier still results in a smooth upper $\mathrm{MgO} / \mathrm{Fe}$ interface (dark field image, (b) and preserves the epitaxial growth (bright field image, c).

(d,e) Cross-sectional views of a 5.5-nm wide, 2-nm thick chromium oxide cluster in a $[\mathrm{MgO}(3 \mathrm{~nm}) / \mathrm{Cr}(0.5 \mathrm{~nm})]_{10}$ stack. In the high angle annular dark field (Z-contrast) image (d), the $\mathrm{Cr}_{2} \mathrm{O}_{3}$ cluster appears as brighter in the darker $\mathrm{MgO}$ matrix. From the bright field image with atomic resolution (e), one may determine the orientational relationship between bcc $\mathrm{MgO}$ and hexagonal $\mathrm{Cr}_{2} \mathrm{O}_{3}: \mathrm{Cr}_{2} \mathrm{O}_{3}[001] \mid \mathrm{MgO}[100]$ and $\mathrm{Cr}_{2} \mathrm{O}_{3}[1 \overline{1} 0] \mid \mathrm{MgO}[001]$ (see Methods). Scale bars, $10 \mathrm{~nm}(\mathbf{b}, \mathbf{c})$ and $2 \mathrm{~nm}(\mathbf{d}, \mathbf{e})$.

the $\mathrm{Cr}_{2} \mathrm{O}_{3}$ particles. As for the small fraction of atoms in a different environment, it is believed to be located at the boundaries of the clusters. Its contribution to absorption could be crudely reproduced using a reference XAS spectrum of $\mathrm{CrO}_{2}$ (ref. 13). This interfacial phase also contributes to the dichroic signal $(20 \%)$. This is consistent with a number of previous works reporting imperfect antiferromagnetic compensation of spins at the surface of $\mathrm{Cr}_{2} \mathrm{O}_{3}$ nanoparticles (see, for example, ref. 14), and with the discovery of a roughness-insensitive magnetization at the surface of bulk $\mathrm{Cr}_{2} \mathrm{O}_{3}$ (ref. 15). 


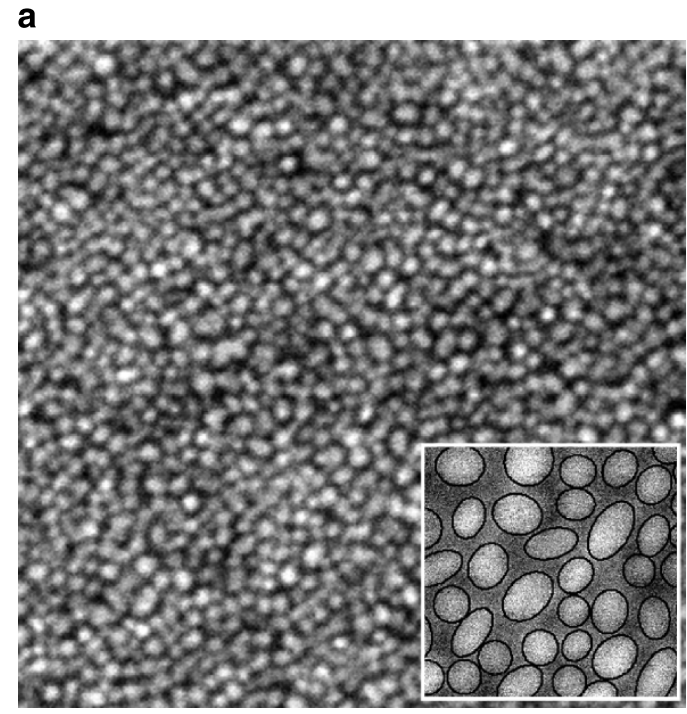

b

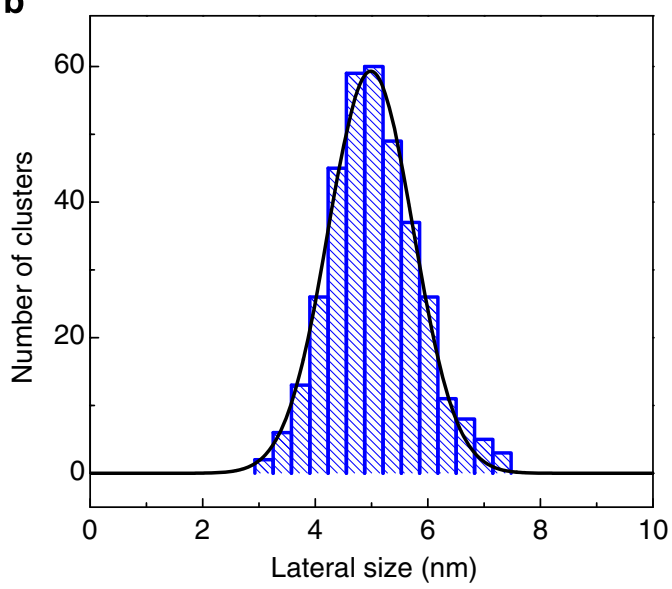

Figure 2 | Chromium nanoclusters on MgO. (a) $200 \times 200 \mathrm{~nm}^{2}$ scanning electron microscopy image of the clusters formed on the lower $\mathrm{MgO}$ tunnel barrier $\left(t_{\mathrm{Cr}}=0.5 \mathrm{~nm}\right)$ before deposition of the upper barrier and annealing. The inset in the lower right corner is an illustration of the treatment performed with the ImageJ software (Methods), in which the clusters are supposed to have ellipsoidal shape. (b) Statistical distribution of the cluster size (square root of surface area) as obtained from an analysis of 460 nanoobjects. The distribution is a gaussian peaked at $5.0 \mathrm{~nm}$, with a full width at half-maximum of $1.5 \mathrm{~nm}$.

Determination of the clusters magnetic moment through TMR. Tunnel magnetoresistance properties of the $\mathrm{Fe} / \mathrm{MgO}: \mathrm{Cr} / \mathrm{Fe}$ devices, very different in shape and amplitude from those of reference $\mathrm{Fe} / \mathrm{MgO} / \mathrm{Fe}(001)$ MTJs (see ref. 9 and Supplementary Fig. 1c), confirm that the oxidized $\mathrm{Cr}$ clusters carry a finite spontaneous magnetic moment (Fig. 4a). Indeed, all of the features of the resistance $(R)$ versus applied magnetic field $(H)$ curves, and especially the characteristic anhysteretic variation of $R$ at large $H$, can be explained within a model of tunnelling between the ferromagnetic $\mathrm{Fe}$ electrodes through superparamagnetic entities $^{16}$. In Supplementary Note 1, we detail the model and its adequacy for fitting the $R(H)$ data and we show that the smallness of the observed TMR effect implies that the conclusions below do not depend on the nature of the tunnelling mechanism (sequential or correlated) effectively involved.

Because of the superparamagnetic behaviour of the clusters assembly, TMR probes the spatial and thermal average of $\cos \theta$, where $\theta$ is the angle of the cluster magnetic moment $\mathbf{m}$ (Fig. 1a) a

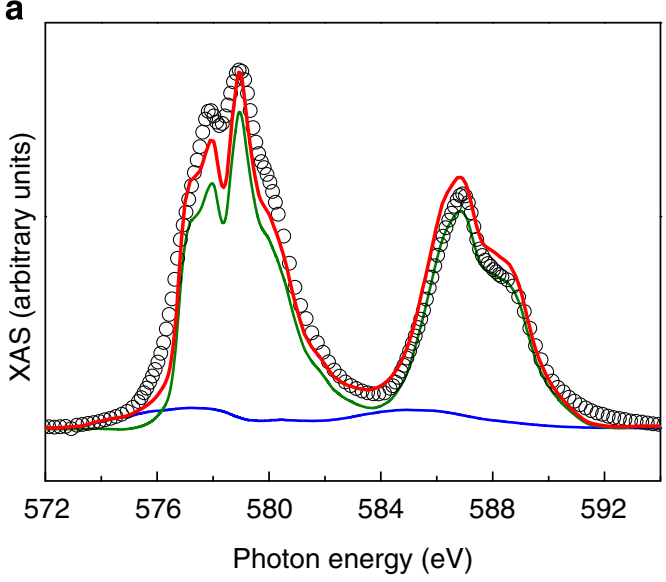

b

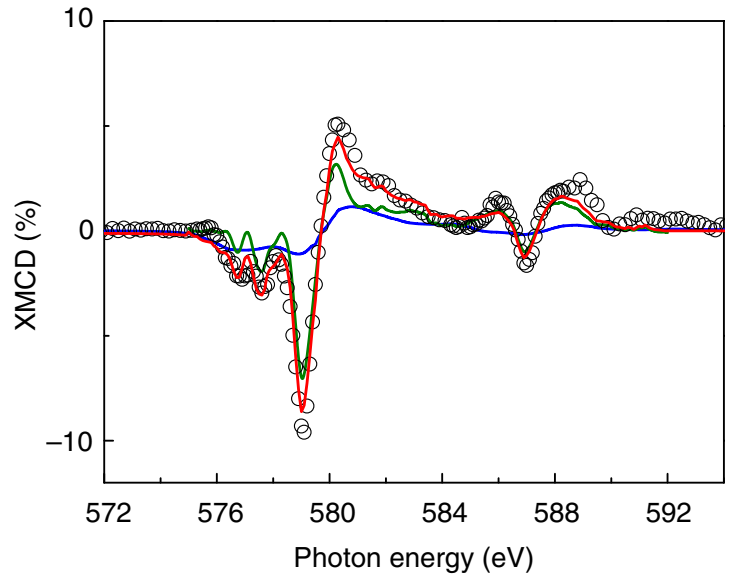

Figure 3 | Oxidized $\mathbf{C r}$ nanoclusters. (a) Isotropic $\mathrm{X}$-ray absorption spectrum at the $\mathrm{Cr} L_{2,3}$ edges of an $\mathrm{MgO}: \mathrm{Cr} / \mathrm{Fe} / \mathrm{MgO}(001)$ sample with nominal $\mathrm{Cr}$ thickness $0.4 \mathrm{~nm}$ (symbols). Data were taken at $2 \mathrm{~K}$. The fit (red line) was obtained assuming $93 \%$ of $\mathrm{Cr}$ atoms in the corundum $\mathrm{Cr}_{2} \mathrm{O}_{3}$ structure (green line) and $7 \%$ in that of $\mathrm{CrO}_{2}$ (blue line). (b) XMCD signal on the same sample as in $\mathbf{a}$. The difference in absorption between left and right circularly polarized lights is normalized to the maximum isotropic absorption at $578.9 \mathrm{eV}$. Chromium atoms in the $\mathrm{Cr}_{2} \mathrm{O}_{3}$ environment contribute $80 \%$ of the integrated signal (same color convention as in $\mathbf{a}$ ).

with respect to the applied magnetic field direction. The $R(H)$ curve follows $\langle\cos \theta\rangle=\mathscr{L}\left(\mu_{0} m H / k_{\mathrm{B}} T\right)$, where $\mu_{0}$ is the magnetic permeability of vacuum, $k_{\mathrm{B}}$ is the Boltzmann constant, and $\mathscr{L}(x)=\operatorname{coth}(x)-1 / x$ is the Langevin function. Hence, at fixed temperature $T$, the aspect of the TMR curve reflects the magnitude of the magnetic moment of the individual nanoparticles, $m$, which can be readily extracted from the data (Fig. $4 \mathrm{a}$ and Supplementary Note 1). For 5-nm wide, 2-nm thick clusters and small bias voltage, $m$ reaches typically $130 \mu_{\mathrm{B}}$ at $6 \mathrm{~K}$, that is about $0.08 \mu_{\mathrm{B}}$ per $\mathrm{Cr}$ atom (of the order of $30 \mathrm{emu} \mathrm{cm}^{-3}$ ). This value, much smaller than the magnetic moment of $\mathrm{Cr}^{3+}$ ions in bulk $\mathrm{Cr}_{2} \mathrm{O}_{3}\left(2.48 \mu_{\mathrm{B}}\right)$ (ref. 17), confirms that the finite magnetization of the chromium oxide clusters originates from spin canting (weak ferromagnetism).

Temperature dependence of the $\mathbf{R}(\mathbf{H})$ curves. A characteristic feature of superparamagnetic assemblies is that their magnetization $M$ scales as $H / T$. This results from the fact that $M$ follows a Langevin function with argument $\left(\mu_{0} m H\right) /\left(k_{\mathrm{B}} T\right)$ and requires that the magnetic dipole moment of each individual 
a

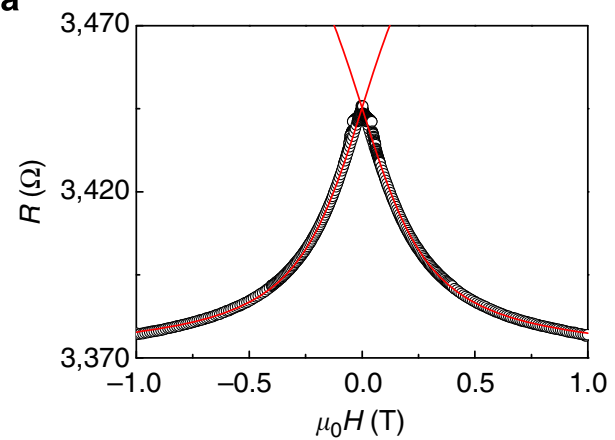

C

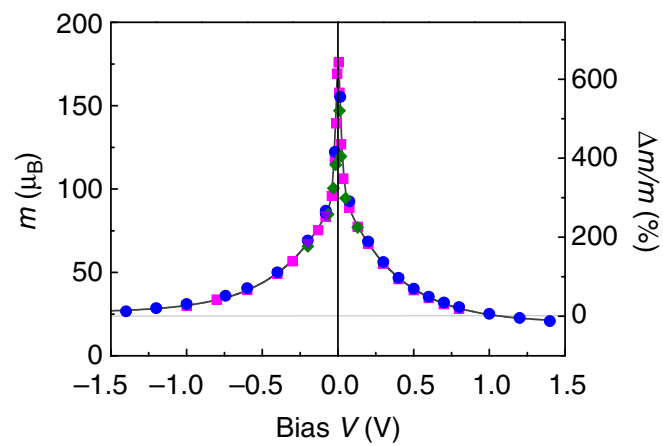

b

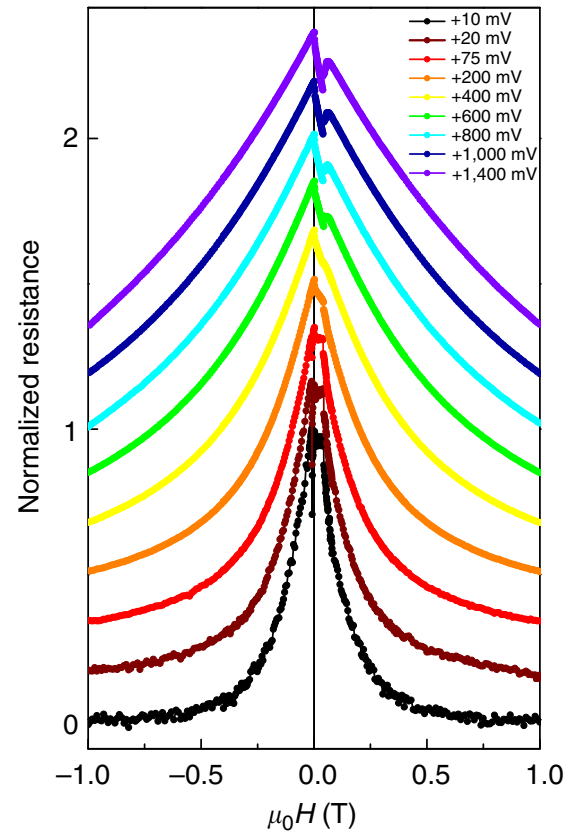

Figure 4 I Tunnel magnetoresistance effects through the chromium oxide clusters. The data shown are for a device with a nominal Cr thickness of $0.3 \mathrm{~nm}$. (a) Typical $R(H)$ curve at $6 \mathrm{~K}$ under a bias voltage $V=130 \mathrm{mV}$ (symbols) and fit to the experimental data assuming sequential tunneling through superparamagnetic clusters with magnetic moment $m=78 \mu_{\mathrm{B}}$ (line). (b) Normalized $R(H)$ curves for increasing bias voltages between $10 \mathrm{mV}$ (black) and $1.4 \mathrm{~V}$ (violet). For the sake of clarity, curves are shifted and only the increasing field branch is shown. (c) Variation of the magnetic moment of the clusters $m$ with the applied voltage $V$ as deduced from $R(H)$ curves at $6 \mathrm{~K}$. Data for four different devices of the same sample are shown with different symbols. The lines are guides to the eyes.

nanomagnet be temperature independent. Figure $5 \mathrm{a}$ shows that the $R(H)$ data recorded on Fe/MgO:Cr/Fe MTJs closely obey such a scaling law.

The superparamagnetic state classically occurs within a limited temperature range. It evolves at lower temperatures towards a blocked state (measurement time shorter than the characteristic time between two flips of the magnetization) and makes a phase transition to the high temperature paramagnetic state at the magnetic Curie point $T_{\mathrm{C}}^{\mathrm{m}}$. Here, the persistence of a still sizable TMR at $250 \mathrm{~K}$ (Fig. 5b,e) indicates that the clusters likely remain magnetically ordered up to nearly room temperature. On the other hand, signs of blocking of the magnetic dipole moments are observed upon lowering of the temperature down to $4 \mathrm{~K}$. For the largest clusters investigated in the present work (mean diameter $\langle d\rangle=7.3 \mathrm{~nm}$ ), this occurs just below $6 \mathrm{~K}$. The signature of this blocking takes the form of a hysteresis in the $R(H)$ loop (Fig. 5c) at magnetic fields much larger than the saturation field of the Fe electrodes (Supplementary Fig. 1b,c). Very much like the magnetization isotherms of assemblies of monodispersive magnetic nanocrystals ${ }^{18}$, the resistance loop then contains both reversible and irreversible contributions.

Electric field dependence of the magnetic moment. The aspect of the $R(H)$ curve strongly depends on the applied bias voltage $V$ (Fig. 4b). This reveals that the cluster magnetic moment is highly sensitive to the electric field $E$. We find that $m$ strongly decreases with increasing voltage (Fig. 4c), the relative change reaching $600 \%$ between $5 \mathrm{mV}$ and $1 \mathrm{~V}$. We note that the variation of $m$ with $V$ is fully reproducible from one MTJ to another if the devices are fabricated under the same conditions. At this point, one needs to rule out the possibility that the decrease of $m$ with $V$ be related to the dispersion in the cluster size: conduction through the smallest nanoparticles, a priori those with the smallest magnetic moments, could be hindered by Coulomb blockade at small bias voltage. This would give rise to an artificially enhanced average magnetic moment as $V$ approaches zero ${ }^{19}$. A couple of strong arguments may be put forward against this scenario. First, the magnetic moment carried by the $\mathrm{Cr}_{2} \mathrm{O}_{3}$ clusters varies with size in a counterintuitive and non-monotonous manner (Fig. 6a) so that the opposite behaviour, (that is, an increase of $m$ with $V$ ), would rather be observed, should Coulomb blockade govern the changes of $m$ (see Supplementary Fig. 2 and Supplementary Note 2). Second, large changes of $m$ with $V$ still occur when the potential energy $\mathrm{eV}$ is much larger than the charging energy of the clusters $e^{2} / C \sim 0.1-0.2 \mathrm{eV}$, where $C \sim 1-2 \mathrm{aF}$ is their estimated capacitance (Supplementary Fig. 2b). We therefore ascribe our observations to a large intrinsic magnetoelectric effect in the $\mathrm{Cr}_{2} \mathrm{O}_{3}$ nanoparticles.

The strongly nonlinear and almost even variation of $m$ with $V$ (Fig. 4c) suggests that quadratic terms in $E$ dominate the magnetoelectric behaviour. This is in agreement with the drastic increase of the quadratic magnetoelectric coefficient predicted in nanoclusters ${ }^{6}$ as well as with the poor symmetry compatibility between weak ferromagnetism and linear magnetoelectric effect ${ }^{20}$. Yet, $m$ does not vary as $V^{2}$. In order to evaluate the sensitivity of magnetization to electric field, we define a voltagedependent effective magnetoelectric coefficient as $\alpha_{\text {eff }}=\frac{\mu_{0}}{V_{\mathrm{Cr}_{2} \mathrm{O}_{3}}} \frac{d m}{d E}$, where $V_{\mathrm{Cr}_{2} \mathrm{O}_{3}}$ is the average volume of the clusters. Under the assumption that the electric field between the metal electrodes is homogeneous, we find $\alpha_{\text {eff }}=6.7 \mathrm{~ns} \mathrm{~m}^{-1}$ at low bias $(10 \mathrm{mV}$, Fig. 4c). This is three orders of magnitude higher than the maximum linear coefficient reported for bulk $\mathrm{Cr}_{2} \mathrm{O}_{3}$, 
a

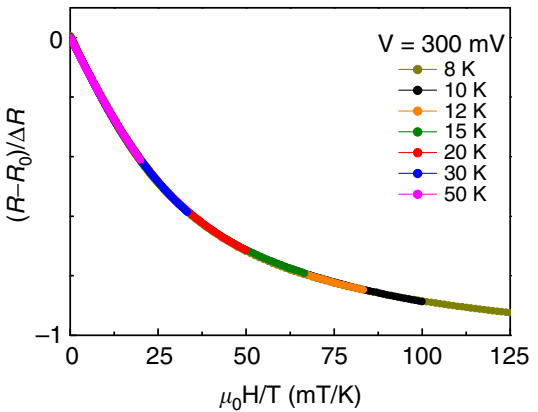

f

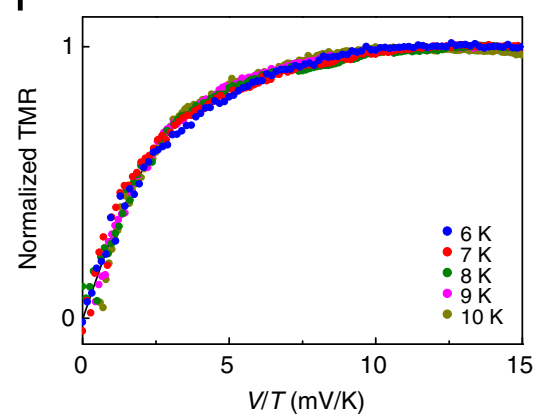

b

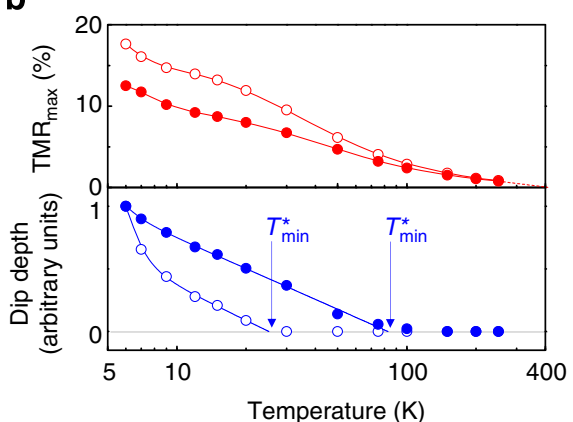

e

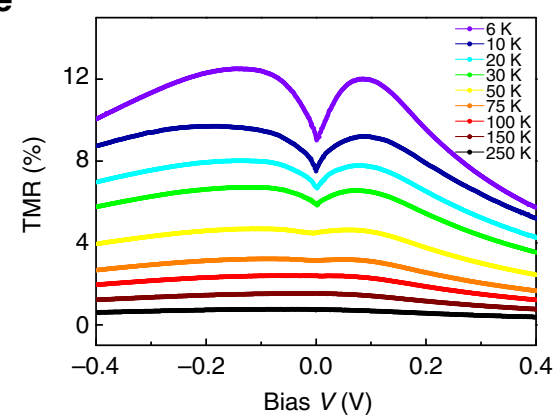

C

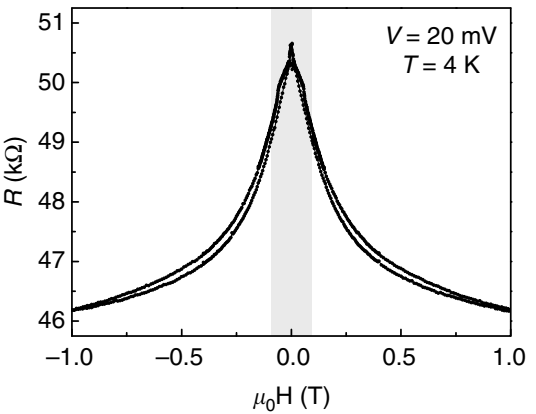

d

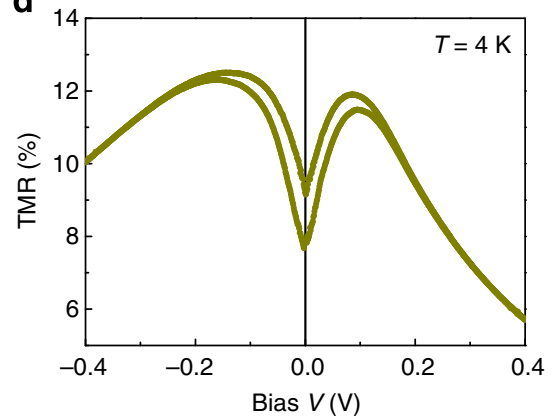

Figure 5 | Thermal behaviour of the superparamagnetic/superparaelectric phase. The data shown are from three different samples with $t_{\mathrm{Cr}}=0.19 \mathrm{~nm}$ $(\mathbf{b}), t_{\mathrm{Cr}}=0.40 \mathrm{~nm}(\mathbf{a}, \mathbf{f})$ and $t_{\mathrm{Cr}}=0.74 \mathrm{~nm}(\mathbf{b}-\mathbf{e})$. The two extreme values correspond, respectively, to the smallest and largest clusters investigated in the present work. (a) $R(H)$ data taken at different temperatures $T$ on decreasing the magnetic field from $1 \mathrm{~T}$ to $0 \mathrm{~T}$ plotted against $H / T$. The data have been normalized to account for the temperature dependence of the magnetoresistance $(\Delta R)$ and zero magnetic field resistance $\left(R_{0}\right)$ of the device. (b) Temperature variations of the maximum TMR ratio (top) and relative depth of the low-bias dip (bottom) for the largest (solid symbols) and smallest clusters (open symbols). (c,d) Hysteretic $R(H)(\mathbf{c})$ and $T M R(V)(\mathbf{d})$ curves obtained at $4 \mathrm{~K}$ for the largest clusters. (e) TMR(V) curves at various temperatures between $6 \mathrm{~K}$ and $250 \mathrm{~K}$ (largest clusters). (f) $T M R(V)$ data at different temperatures plotted against $V / T$. The raw data have been normalized to eliminate the temperature dependence of the depth of the low-bias dip.

$\alpha=4.17 \mathrm{psm}^{-1}$ at $263 \mathrm{~K}$ (ref. 5). Note that $\alpha_{\text {eff }}$ goes down to $29 \mathrm{ps} \mathrm{m}^{-1}$ at $1 \mathrm{~V}$, which is still large compared with bulk.

Influence of the clusters size on the magnetization. To investigate the influence of the size of the nanoparticles, we grew samples with a gradient in the nominal thickness of chromium such that $0.1 \mathrm{~nm}<t_{\mathrm{Cr}}<0.8 \mathrm{~nm}$. Along the gradient, the height $(h=2 \mathrm{~nm})$ and the density $\left(\sigma=2 \times 10^{4} \mu \mathrm{m}^{-2}\right)$ of the clusters are nearly constant (ref. 10 and Methods) and, consequently, the inplane surface area and the volume of the clusters are proportional to $t_{\mathrm{Cr}}$-see Fig. 6 for the correspondence between $t_{\mathrm{Cr}}$ and cluster size. Figure 6a shows the variation of $m$ with $t_{\mathrm{Cr}}$ as extracted from magnetotransport measurements taken on different MTJs along the gradient. As already mentioned before, $m$ does not increase monotonously with the size of the particles. Instead, it exhibits a broad maximum at $t_{\mathrm{Cr}} \sim 0.35 \mathrm{~nm}$. According to what precedes, $m / t_{\mathrm{Cr}}$ is proportional to the magnetic moment per $\mathrm{Cr}$ atom, that is, to magnetization. As illustrated in Fig. 6b, this ratio diminishes exponentially as the size of the nanoparticles increases. The same behaviour with a characteristic decay constant of $\lambda_{\mathrm{m}}=(0.36 \pm 0.01) \mathrm{nm}$ is found whatever the applied voltage and the temperature.

In the epitaxial growth of quantum dots, following either the Volmer-Weber or the Stranski-Krastanov modes, the introduction of dislocations ${ }^{21}$ and the elastic relaxation at the dot edges ${ }^{22}$ often yield an exponential relaxation of stress with increasing layer thickness. The epitaxial relationship between the $\mathrm{Cr}_{2} \mathrm{O}_{3}$ inclusions and the surrounding $\mathrm{MgO}$ matrix implies nominal in-plane compressive strains of $-7 \%$ along the $c$ axis of the corundum structure, and of $-18 \%$ perpendicular to it (Methods). In epitaxial clusters, such a large lattice mismatch usually starts relaxing from the beginning of growth ${ }^{22}$. For $t_{\mathrm{Cr}}=0.4 \mathrm{~nm}$, photoluminescence spectra (Fig. 7a) taken on a large ensemble of nanoparticles are indicative of average compressive stress in excess of $6 \mathrm{GPa}$ and, for $t_{\mathrm{Cr}}=0.5 \mathrm{~nm}$, observations of six individual clusters by scanning transmission electron microscopy with atomic resolution (Figs 1e,7b, Supplementary Table 1) reveal an actual strain along $c$ in the range of -3 to $-6 \%$, consistent with a partial structural relaxation. On the basis of these considerations, we consider the exponential decay of $m / t_{\mathrm{Cr}}$ with $t_{\mathrm{Cr}}$ as a strong indication that compressive epitaxial strain is responsible for the finite magnetization of the chromium oxide clusters. This is consistent with recent experimental works ${ }^{23,24}$ reporting a remnant magnetic moment in granular $\mathrm{Cr}_{2} \mathrm{O}_{3}$ arising from magneto-elastic effects. Note that, following the model of Glinchuk et al. ${ }^{6}$, non-zero magneto-elastic coefficients can explain the drastic enhancement of magnetoelectric properties we observe in strained $\mathrm{Cr}_{2} \mathrm{O}_{3}$ clusters.

Voltage dependence of the TMR ratio. The voltage dependence of the TMR ratio defined as $1-R(H=1 T) / R(H=0)$ shows remarkable low-bias features (Fig. 8), up to about $80 \mathrm{~K}$ (Fig. 5b,e). In addition to the overall bell shape usually seen for $\mathrm{Fe} / \mathrm{MgO} / \mathrm{Fe}$ junctions ${ }^{9}$, we observe for the $\mathrm{Fe} / \mathrm{MgO}: \mathrm{Cr} / \mathrm{Fe}$ devices a dip in the $(-80 \mathrm{mV},+80 \mathrm{mV})$ voltage range. Similar features have already been reported by Yang and coworkers ${ }^{25}$ for TMR stacks with embedded nanometric clusters made of a CoFe metallic alloy. The authors have attributed the dips to a Kondo effect in the 


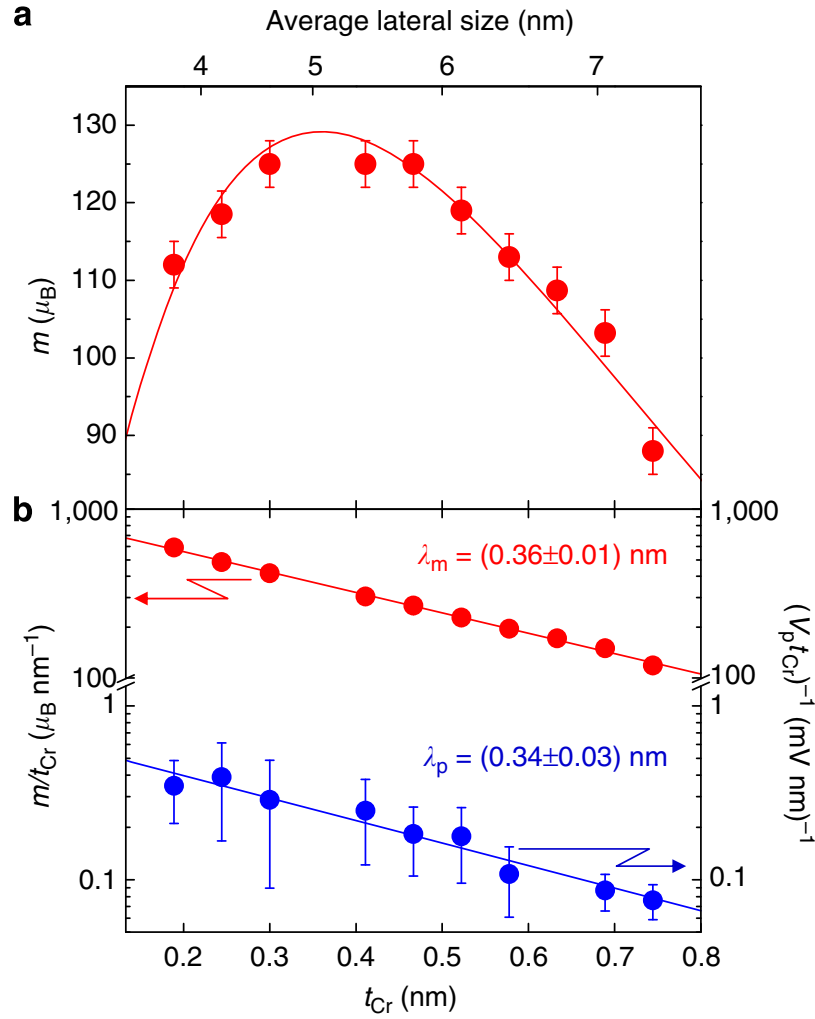

Figure 6 | Influence of the size of the chromium oxide clusters on the ferroic order parameters. (a) Average magnetic moment of the clusters $m$, at $6 \mathrm{~K}$ and under $-10 \mathrm{mV}$ bias voltage, as a function of the nominal thickness of chromium deposited $t_{\mathrm{Cr}_{r}}$ (bottom scale) or average cluster size (top scale). The line is a fit according to $m\left(t_{C_{r}}\right)=m_{0} \exp \left(-t_{C r} / \lambda_{m}\right) t_{C r}$ with $m_{0}=966 \mu_{\mathrm{B}}$ and $\lambda_{\mathrm{m}}=0.36 \mathrm{~nm}$. (b) Semi-logarithmic plot of $\mathrm{m} / \mathrm{t}_{\mathrm{Cr}}$ (magnetization, red symbols) and $\left(V_{p} t_{C r}\right)^{-1}$ (electric polarization, blue symbols) versus $t_{\mathrm{Cr}}$ and linear fits (lines) to the experimental data. The error bars are derived from the standard errors of the least-squares fits performed to extract $m$ and $V_{p}$ from $R(H)$ and $T M R(V)$ data curves.

ferromagnetic clusters that opens an additional spin-independent conduction channel at low voltage. Unlike in ref. 25, we do not observe the increase in conductance near zero bias voltage (Supplementary Fig. 2c) indicative of a Kondo resonance 26. Therefore, we rule out such an explanation here. In the following, we propose an explanation in terms of ferroelectricity.

\section{Discussion}

Theoretical works on MTJs with ferroelectric insulating barrier ${ }^{27}$ predict that the orientation of the electric polarisation can influence the electron density of states at the ferromagnetic/ insulator interfaces and modify the asymmetry between majority and minority electrons. Such modification implies a change in the spin polarisation of the tunnelling electrons and should therefore show up in TMR properties. Consistently, Garcia et al. ${ }^{28}$ have observed an abrupt change in TMR on reversal of the polarisation of $\mathrm{a} \mathrm{BaTiO}_{3}$ tunnel barrier. Here no such abrupt change is observed but, in the low-bias range, the TMR curve may be fitted to the equation $\operatorname{TMR}(V)=T M R_{0}+T M R_{1} \times\left|\mathscr{L}\left(V / V_{\mathrm{p}}\right)\right|$ (see inset in Fig. 8), where $T M R_{0}$ and $T M R_{1}$ are constants, $V_{\mathrm{p}}$ is a characteristic voltage value, and $\mathscr{L}$ is again the Langevin function. We therefore argue that the $\mathrm{Cr}_{2} \mathrm{O}_{3}$ clusters are ferroelectrically ordered and that they behave as superparaelectrics.

The concept of superparaelectricity was defined many years ago $^{29}$ by analogy with superparamagnetism. It should become increasingly relevant in the search of ferroelectricity at the nanoscale ${ }^{30}$ and, indeed, a recent work on $\mathrm{BaTiO}_{3}$ nanocubes also reports a possible signature of this $\mathrm{effect}^{31}$. In the superparaelectric state, the permanent electric dipole moments of small enough ferroelectric particles fluctuate in direction because thermal energy exceeds the energy barrier against polarisation rotation. Applying an electric field $\mathbf{E}$ to an assembly of such particles orients their electric dipoles preferentially in the direction of $\mathbf{E}$ and the resulting total electric polarisation follows a Langevin law. The dip in the $\operatorname{TMR}(V)$ curve reflects such a behaviour. The way it changes with temperature also ascertains the superparaelectric nature of the $\mathrm{Cr}$ oxide clusters. Indeed, in the temperature range where the bell-shaped background TMR signal evolves little in shape, the low-bias part of the $T M R(V)$ curves obeys the expected $V / T$ scaling (Fig. 5f), which is the pendant of the $H / T$ scaling in superparamagnetism (Fig. 5a).

Taking the electric field as homogeneous through the entire $\mathrm{MgO} / \mathrm{Cr}_{2} \mathrm{O}_{3} / \mathrm{MgO}$ stack of total thickness $d \sim 6 \mathrm{~nm}$ $(E=V / d)$, one can evaluate the average electric dipole $p$ of the $\mathrm{Cr}_{2} \mathrm{O}_{3}$ clusters from $(V) /\left(V_{\mathrm{p}}\right)=(p E) /\left(k_{\mathrm{B}} T\right)$. For $t_{\mathrm{Cr}}=0.3 \mathrm{~nm}$ (Fig. 8), we get $p=7.4 \times 10^{-29} \mathrm{Cm}$. The corresponding electric polarization $P$ is of the order of $0.22 \mu \mathrm{C} \mathrm{cm}^{-2}$. It is small compared to that of the archetypical material $\mathrm{BiFeO}_{3}$ (ref. 32), but of the same order of magnitude as those of improper ferroelectrics where the spontaneous polarization is a secondary-order parameter induced by lattice distortion ${ }^{33,34}$. It is also of the same order of magnitude as the value of $0.585 \mu \mathrm{Ccm}^{-2}$ calculated by Mostovoy et al. ${ }^{35}$ for $\mathrm{Cr}_{2} \mathrm{O}_{3}$ in a hypothetical multiferroic state realized by reversing one out of four spins in the rhombohedral unit cell. Such a low polarisation value favours the occurrence of superparaelectricity. First, it makes the electrostatic interactions between clusters weak and thus prevents the formation of a globally ordered state ${ }^{36}$ Second, it implies a low energetic barrier against polarisation rotation, which is an even stronger requirement. This energy barrier scales as $P^{2}$ (refs 30,36) and its size is of the order of the energy $W_{\mathrm{C}}$ of a ferroelectric capacitor with volume $V: W_{\mathrm{C}}=(1) /(2) V P^{2} / \epsilon \epsilon_{0}$ (ref. 30), where $\epsilon_{0}$ is the permittivity of vacuum. For $\mathrm{Cr}$ oxide clusters with dielectric constant $\varepsilon \sim 11$ (at least), volume $V=35 \mathrm{~nm}^{3}$, and polarisation $P=0.22 \mu \mathrm{Ccm}-2$ $\left(t_{\mathrm{Cr}}=0.3 \mathrm{~nm}\right), W_{\mathrm{C}}$ amounts to $5.4 \mathrm{meV}$, which is equivalent to $60 \mathrm{~K}$. We note that this is the right order of magnitude for observing superparaelectricity down to temperatures of a few Kelvin.

By analogy with superparamagnetism, one expects that the superparaelectric state be preceded by a blocked state at lower temperatures and, indeed, a sign of the blocking of (some of) the electric dipole moments in the form of a hysteresis in the $T M R(V)$ data is observed for the largest clusters, below $6 \mathrm{~K}$ (Fig. 5d). On the high temperature side, the superparaelectric state should be followed by a paraelectric state beyond the electric Curie point $T_{C}^{p}$. We note that the low-bias dip in the $\operatorname{TMR}(V)$ curve, which we consider as a signature of the electric ordering, disappears beyond a temperature which increases from $T_{\text {min }}^{*} \sim 20 \mathrm{~K}$ for the smallest clusters considered in the present study (mean diameter $\langle d\rangle=3.7 \mathrm{~nm})$ to $T_{\max }^{*} \sim 80 \mathrm{~K}$ for the largest ones $(\langle d\rangle=7.3$ $\mathrm{nm})$, see Fig. 5b. However, since TMR is an indirect probe of the ferroelectricity of the clusters, $T^{*}$ should only be considered, at best, as a lower bound for $T_{C}^{p}$.

A strong and final argument for the occurrence of ferroelectric ordering in the clusters is brought by the size dependence of $V_{\mathrm{p}}$. Just as $m / t_{\mathrm{Cr}}$ is a measure of the clusters magnetization, $\left(V_{\mathrm{p}} t_{\mathrm{Cr}}\right)^{-1}$ should be a measure of their electric polarization. As seen in Fig. 6b, $\left(V_{\mathrm{p}} t_{\mathrm{Cr}}\right)^{-1}$ also decreases exponentially with increasing $t_{\mathrm{Cr}}$, with a characteristic decay constant 
a

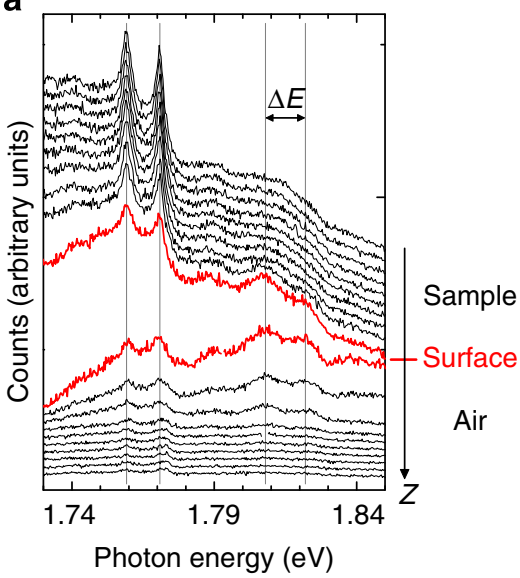

b

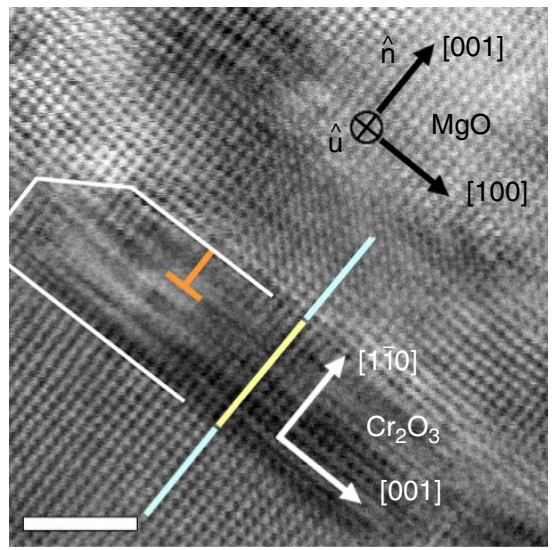

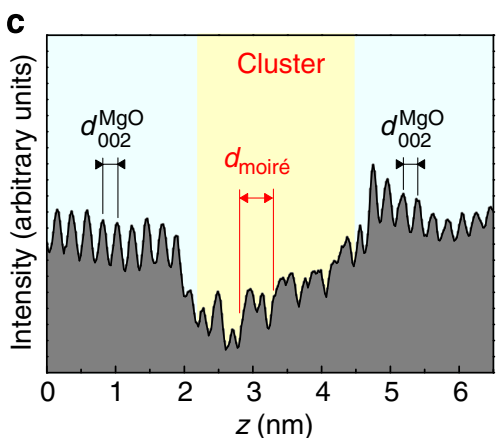

Figure 7 | Evidence of compressive strain in the chromium oxide clusters. (a) Micro-photoluminescence spectra obtained at different focalisation height on an $\mathrm{MgO}: \mathrm{Cr} / \mathrm{Fe} / \mathrm{MgO}(001)$ stack with $t_{\mathrm{cr}}=0.4 \mathrm{~nm}$. A spectrum was recorded every $0.5 \mu \mathrm{m}$, from $z=+5.5 \mu \mathrm{m}$ above the nominal position of the sample surface (bottom) to $z=-4.5 \mu \mathrm{m}$ below (top). The high energy peaks (1.81-1.82 eV), the intensity of which is the strongest when the laser beam is focused close to the sample surface (thick red lines), are attributed to emission from $\mathrm{Cr}^{3+}$ ions in the $\mathrm{Cr}_{2} \mathrm{O}_{3}$ clusters. The energy difference between these $R_{1}$ and $R_{2}$ lines ( $\Delta E=13 \mathrm{meV}$ ) is consistent with an average compressive stress in excess of $6 \mathrm{GPa}$ (Methods). (b) High resolution image of an exceptionally large cluster ( $10 \mathrm{~nm}$ wide, $2.3 \mathrm{~nm}$ thick) in an $[\mathrm{MgO}(3 \mathrm{~nm}) / \mathrm{Cr}(0.5 \mathrm{~nm})]_{10}$ multilayer stack. A dislocation is observed close to its upper surface (orange sign). Scale bar, $2 \mathrm{~nm}$. (c) Intensity profile along the coloured line in $\mathbf{b}$, which is parallel to the normal to the film. The $d_{\text {moiré }}=0.45 \mathrm{~nm}$ periodicity of the contrast in the cluster (yellow) region corresponds to parallel moire fringes formed by the overlapping of (200) $\mathrm{MgO}$ planes with spacing $d_{002}^{\mathrm{MgO}}=0.215 \mathrm{~nm}$ and $(300) \mathrm{Cr}_{2} \mathrm{O}_{3}$ planes with spacing $d_{300}^{\mathrm{Cr}_{2} \mathrm{O}_{3}}=0.145 \mathrm{~nm},\left(d_{\text {moire }}\right)^{-1}=\left(d_{300}^{\mathrm{Cr}_{2} \mathrm{O}_{3}}\right)^{-1}-\left(d_{002}^{\mathrm{MgO}}\right)^{-1}$.

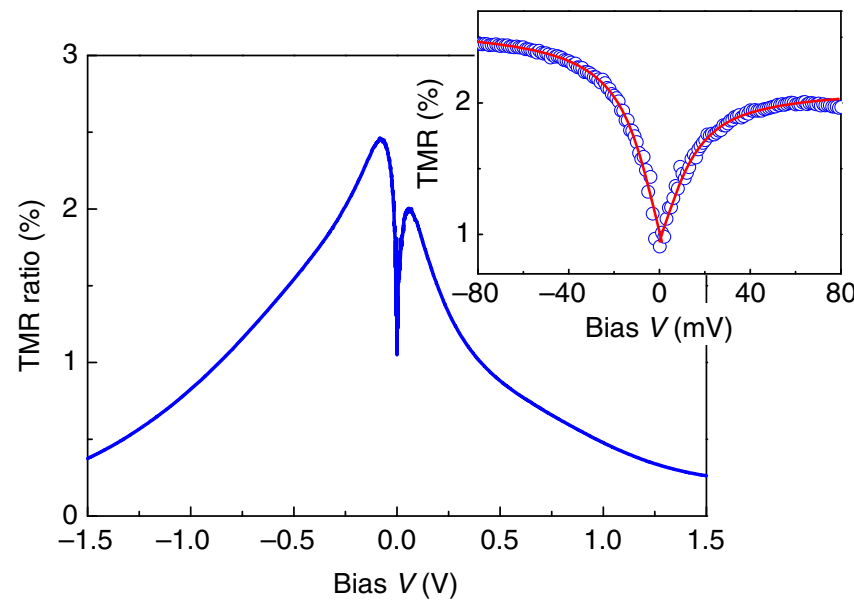

Figure 8 | Bias dependence of TMR. Variation of the TMR ratio with the applied voltage, at $6 \mathrm{~K}$, for a device with $t_{\mathrm{Cr}}=0.3 \mathrm{~nm}$. The inset shows a zoom in the low voltage range of the $T M R(V)$ curve (symbols) and fits of the experimental data (lines) to the expression $T M R(V)=T M R_{0}+T M R_{1} \times\left|\mathscr{L}\left(V / V_{p}\right)\right|$, with $T M R_{0}=0.99 \%$ and $V_{\mathrm{p}}=7.1 \mathrm{mV}$. To account for the usual asymmetry of the bias dependence of TMR in Fe/MgO-based magnetic tunnel junctions ${ }^{9}$, different values of $T M R_{1}$ were allowed for positive (1.14\%) and negative (1.61\%) voltages. $\lambda_{\mathrm{p}}=(0.34 \pm 0.03) \mathrm{nm}$ very close to that of the magnetization, $\lambda_{\mathrm{m}}$. There is therefore experimental evidence that the two dipoles, $p$ and $m$, share a common origin: epitaxial strain drives the value of the magnetic dipole $m$ as well as that of the electric dipole $p$. The occurrence of a permanent electric polarisation under stress in paraelectric materials with non-zero coupling between lattice and electric order has indeed been predicted ${ }^{6,7,37}$ and observed ${ }^{8}$ in other systems. We thus conclude that the $\mathrm{Cr}_{2} \mathrm{O}_{3}$ particles exhibit both a (weak) ferromagnetic order and a ferroelectric order.

In summary, the ferroic properties of $\mathrm{Cr}_{2} \mathrm{O}_{3}$ are drastically changed at the nanoscale. Epitaxial $\mathrm{Cr}_{2} \mathrm{O}_{3}$ nanoclusters imprisoned in a single crystalline $\mathrm{MgO}$ matrix experience large compressive strain that makes an original superparamagnetic/ superparaelectric phase appear. The clusters also exhibit large magnetoelectric effects with effective coefficients in the $\mathrm{ns}^{-1}$ range, which compare with those obtained in piezoelectric/ piezomagnetic nanocomposites ${ }^{38}$. Combined with superparamagnetism, these large effects imply a very strong electric field dependence of the magnetic susceptibly or electroinductance effect. It can be regarded as the symmetric effect of the intensively studied magneto-capacitance phenomenon ${ }^{33}$ and could find important applications outside the field of spintronics, for instance, to build devices with electric field-controlled inductance. 


\section{Methods}

Thin film growth and MTJ fabrication. The epitaxial (001) oriented heterostructures were grown by molecular beam epitaxy on (001) $\mathrm{MgO}$ substrates. The multilayers were deposited at $470 \mathrm{~K}$, and subsequently annealed at $720 \mathrm{~K}$ for one hour in ultrahigh vacuum. The final stacking sequence is $\mathrm{Pt}(2) / \mathrm{Co}(15) / \mathrm{Fe}(5) /$ $\mathrm{MgO}(2.5$ or 3$) / \mathrm{Cr}\left(t_{\mathrm{Cr}}\right) / \mathrm{MgO}(2.5) / \mathrm{Fe}(20) / \mathrm{MgO}$, where thicknesses are given in $\mathrm{nm}$. The top $\mathrm{Fe} / \mathrm{Co}$ bilayer is the magnetically harder electrode. In some cases, a gradient in $\mathrm{Cr}$ thickness was realized by moving progressively a shutter across the sample during deposition. Tunnel junction devices with diameter of 8-30 $\mu \mathrm{m}$ were patterned using standard microfabrication techniques, including $U V$ lithography, Ar ion etching, encapsulation with $\mathrm{Si}$ oxide and metal $(\mathrm{Al} / \mathrm{Au})$ liftoff to realize the top electrical contact.

Scanning electron microscopy observations. Scanning electron microscopy was used to observe the $\mathrm{Cr}$ clusters formed on the $\mathrm{MgO} / \mathrm{Fe} / \mathrm{MgO}(001)$ surface before being covered with $\mathrm{MgO}$ and annealed. An example of micrograph obtained for $t_{\mathrm{Cr}}=0.5 \mathrm{~nm}$, shortly after deposition, is shown in Fig. 2, together with the corresponding distribution of cluster lateral size as deduced from an image analysis performed with the ImageJ software. The scattering in the size of the clusters is rather moderate: the diameter distribution is a gaussian peaked at $5.0 \mathrm{~nm}$, with a full width at half-maximum of $1.5 \mathrm{~nm}$. Images taken along a $\mathrm{Cr}$ wedge $0.2 \mathrm{~nm} \leq t_{\mathrm{Cr}} \leq 0.6 \mathrm{~nm}$ indicate that, within $5 \%$, the cluster density remains constant (approximately $2 \times 10^{4} \mu \mathrm{m}^{-2}$ ) as the amount of metal deposited increases, in agreement with previous observations by Koda et al. ${ }^{10}$

Magnetotransport experiments. Measurements were carried out using a conventional $D C$ 4-wire technique, with the positive reference for voltage taken at the top $(\mathrm{Fe} / \mathrm{Co})$ electrode and the external magnetic field applied along the [100] easy direction of magnetization of the bcc Fe layers. The $T M R(V)$ curves were obtained by combining current-voltage characteristics measured successively, with external magnetic fields of $1 \mathrm{~T}$ and $0 \mathrm{~T}$. No significant difference in the magnetotransport data could be observed whether or not a magnetic field and/or an electric field was applied during cooling of the sample from room temperature down to $6 \mathrm{~K}$.

X-ray absorption experiments. XAS and XMCD measurements were performed at $2 \mathrm{~K}$ on a dedicated $\mathrm{MgO}: \mathrm{Cr} / \mathrm{Fe} / \mathrm{MgO}(001)$ sample with $t_{\mathrm{Cr}}=0.4 \mathrm{~nm}$, using the cryomagnetic endstation available at the DEIMOS beamline of SOLEIL synchrotron. The absorption was measured at the $L_{2,3}$ edges of $\mathrm{Cr}$ through the total electron yield with a large beam size of about $800 \times 800 \mu \mathrm{m}^{2}$ and a low photon flux so as to avoid charging electrically the sample. The presence, below the $\mathrm{MgO}$ layer containing the clusters, of a 20 -nm thick metal film much thicker than the light penetration depth (a few nanometres) excluded any contribution to the recorded spectra from the $\mathrm{Cr}^{3+}$ ionic impurities usually present in bulk $\mathrm{MgO}$ substrates ${ }^{39}$. The XMCD signal was obtained by combining XAS spectra recorded with left and right circularly polarized lights and applied magnetic fields of $+6 \mathrm{~T}$ and $-6 \mathrm{~T}$. Experimental data were fitted to a linear superposition of reference spectra found in the literature for bulk $\alpha-\mathrm{Cr}_{2} \mathrm{O}_{3}$ (XAS) ${ }^{40}, \mathrm{Cr}^{3+}$ ions in ruby $\alpha-\mathrm{Al}_{2} \mathrm{O}_{3}: \mathrm{Cr}^{3+}$ $(\mathrm{XMCD})^{12}$ and a thick $\mathrm{CrO}_{2}$ film (XAS and XMCD) ${ }^{13}$.

Scanning transmission electron microscopy observations. STEM in cross section was used to characterize the morphology of the $\mathrm{MgO}$ tunnel barrier, visualize the embedded clusters and determine their epitaxial relationship and the related lattice mismatch with the matrix material. STEM images show that, despite the inclusion of $\mathrm{Cr}$, the $\mathrm{MgO}$ barrier has uniform thickness and smooth interfaces (Fig. 1b). The clusters have almost equal height $(h=2 \mathrm{~nm})$ while their lateral dimensions are within the $4-8 \mathrm{~nm}$ range for $t_{\mathrm{Cr}}=0.5 \mathrm{~nm}$. Atomic resolution pictures reveal that crystalline planes are continuous throughout the $\mathrm{MgO}$ matrix and the clusters (Figs 1f,7b), meaning that the latter are still epitaxial after being covered and annealed/oxidized. No clear micro-diffraction patterns could be obtained on such clusters. Yet, atomic planes parallel to both the normal to the film $\hat{\mathbf{n}}$ and the zone axis $\hat{\mathbf{u}}$ ([010] direction of $\mathrm{MgO})$ in Fig. $7 \mathrm{~b}$ have $(0.218 \pm 0.002) \mathrm{nm}$ periodicity, which is close to the distance between (006) planes in bulk $\mathrm{Cr}_{2} \mathrm{O}_{3}$, $d_{300}^{\mathrm{Cr}_{2} \mathrm{O}_{3}}=0.227 \mathrm{~nm}$ (here Miller indices for chromium oxide refer to the hexagonal unit cell). Moreover, the distance between atomic planes perpendicular to $\hat{\mathbf{n}}$ $((0.145 \pm 0.004) \mathrm{nm}$, Fig. $7 \mathrm{c})$ matches that between (300) planes in bulk, $d_{300}^{\mathrm{C}_{2} \mathrm{O}_{3}}=0.143 \mathrm{~nm}$. This shows that the $c$ axis of the corundum structure lies in the plane of the layers, parallel to the [100] direction of $\mathrm{MgO}$. Overall, the orientational relationship between the matrix and cluster materials can be summarized as follows: $\mathrm{Cr}_{2} \mathrm{O}_{3}[001]|| \mathrm{MgO}[100]$ and $\mathrm{Cr}_{2} \mathrm{O}_{3}[1 \mathrm{1} 0] \| \mathrm{MgO}[001]$. This is fully consistent with i) the epitaxial growth of bcc $\mathrm{Cr}(001)$ on $\mathrm{MgO}(001)$ with the well-known orientational relationship $\mathrm{Cr}[100] \| \mathrm{MgO}[110]$ and ii) the fact that $\mathrm{Cr}_{2} \mathrm{O}_{3}$ formed by oxidation of metallic $\mathrm{Cr}$ usually obeys $\mathrm{Cr}_{2} \mathrm{O}_{3}[001] \| \mathrm{Cr}[110]$ (refs 41,42 ), and sometimes also $\mathrm{Cr}_{2} \mathrm{O}_{3}[1 \overline{1} 0]|| \mathrm{Cr}[001]$ (ref. 42). From the orientational relationship, the lattice mismatch between the matrix and cluster materials can be estimated: $1-d_{300}^{\mathrm{Cr}_{2} \mathrm{O}_{3}} / d_{002}^{\mathrm{MgO}}=-7 \%$ along the $c$ axis direction, and $1-d_{300}^{\mathrm{Cr}_{2} \mathrm{O}_{3}} / d_{002}^{\mathrm{MgO}}=-18 \%$ along the in-plane direction that is perpendicular to $c$. Cr oxide nanoparticles imprisoned in the $\mathrm{Mg}$ oxide matrix are then bound to be under strong in-plane biaxial compressive stress. Because of stress relaxation through the introduction of dislocations, such as the one which is visible in Fig. $7 \mathrm{~b}$, and deformation at the cluster edges, the actual in-plane strain is, however, reduced. In the cluster of Fig. $7 \mathrm{~b}$, the strain along $c$ amounts to $-(4 \pm 1) \%$, which, with a Young modulus of $315 \mathrm{GPa}$ (ref. 43), corresponds to a large stress of $-(13 \pm 3) \mathrm{GPa}$. All the clusters that were observed by STEM showed similar compressive in-plane strains (Supplementary Table 1). Along $\hat{\mathbf{n}}$, the distance between atomic planes is measured with too large an error bar that prevents strain evaluation.

Micro-photoluminescence. The photoluminescence and Raman spectra of $\mathrm{Cr}^{3+}$ ions are known to be sensitive to the hydrostatic or biaxial stress in corundum structures $^{44-46}$. More precisely, Nychka et al. ${ }^{44}$ and Selcuk et al. ${ }^{45}$ showed that the $R_{1}$ and $R_{2}$ luminescence lines have different piezo-spectroscopic coefficients so that the energy difference between them, $\Delta E$, which is $4 \mathrm{meV}$ in unstressed samples, increases with increasing compressive stress. It was demonstrated experimentally ${ }^{44}$ that, up to a biaxial stress of $6 \mathrm{GPa}, \Delta E$ is linear in stress and increases by about $0.1 \mathrm{meV}$ per GPa. Above $6 \mathrm{GPa}$, no systematic measurements were carried out but a nonlinear behaviour is expected ${ }^{45}$. To further ascertain the presence of strain in our chromium oxide clusters, we therefore recorded micro-photoluminescence spectra in a backscattering configuration using a laser beam at $2.33 \mathrm{eV}$ with a Rayleigh length of about $1 \mu \mathrm{m}$. To avoid light absorption, we used a dedicated sample $\left(t_{\mathrm{Cr}}=0.4 \mathrm{~nm}\right)$ without top electrode but with a $20 \mathrm{~nm}$ bottom Fe electrode acting as a semi-transparent mirror.

Spectra recorded at varying focalisation height $z$ of the laser beam (Fig. 7a) show two close peaks in the 1.76-1.77 eV energy range, irrespective of $z$. Since these are also present in the spectra of bare $\mathrm{MgO}$ substrates, one can ascertain that they arise from impurities which are systematically present in bulk $\mathrm{MgO}$, with concentrations in the tens of p.p.m. range ${ }^{39}$. The observed peaks coincide quite well with the R-line $(1.776 \mathrm{eV})$ from $\mathrm{Cr}^{3+}$ ions in octahedral symmetry sites and the $\mathrm{N}$-lines $(1.773$ and $1.762 \mathrm{eV})$ associated with $\mathrm{Cr}^{3+}$ ions in tetragonal symmetry sites $^{47}$. When the beam is focused on the surface $(z \sim 0)$, the luminescence spectrum exhibits two extra peaks at higher energy $(1.81-1.82 \mathrm{eV})$, which are interpreted as the $\mathrm{R}_{1}$ and $\mathrm{R}_{2}$ emission lines of $\mathrm{Cr}^{3+}$ ions in the chromium oxide clusters. The energy difference $\Delta E$ between them $(13 \mathrm{meV})$ is much larger than in unstrained materials and exceeds the value associated with the $6 \mathrm{GPa}$ maximum stress investigated in refs 44,45 . This indicates that an average stress in excess of $6 \mathrm{GPa}$ is present in the clusters, in qualitative agreement with TEM observations.

\section{References}

1. Spalding, N. A. \& Fiebig, M. The renaissance of magnetoelectric multiferroics. Science 309, 391-392 (2005).

2. Ramesh, R. \& Spalding, N. A. Multiferroics: progress and prospects in thin films. Nat. Mater. 6, 21-29 (2007).

3. Curie, P. Sur la symétrie dans les phénomènes physiques, symétrie d'un champ électrique et d'un champ magnétique. J. Phys. 3(Ser. III): 393-415 (1894).

4. Eerenstein, W., Mathur, N. D. \& Scott, J. F. Multiferroic and magnetoelectric materials. Nature 442, 759-765 (2006).

5. Astrov, D. N. The magnetoelectric effect in antiferromagnetics. Sov. Phys. JETP 11, 708-709 (1960).

6. Glinchuk, M. D., Eliseev, E. A., Morozovska, A. N. \& Blinc, R. Giant magnetoelectric effect induced by intrinsic surface stress in ferroic nanorods. Phys. Rev. B 77, 024106 (2008).

7. Fennie, C. J. \& Rabe, K. M. Magnetic and electric control in epitaxial $\mathrm{EuTiO}_{3}$ from first principles. Phys. Rev. Lett. 97, 267602 (2006).

8. Lee, J. H. et al. A strong ferroelectric ferromagnet created by means of spinlattice coupling. Nature 466, 954-959 (2010).

9. Yuasa, S., Nagahama, T., Fukushima, A., Susuki, Y. \& Ando, K. Giant roomtemperature magnetoresistance in single-crystal $\mathrm{Fe} / \mathrm{MgO} / \mathrm{Fe}$ magnetic tunnel junctions. Nat. Mater. 3, 868-871 (2004).

10. Koda, T., Mitani, S., Mizogushi, M. \& Takanashi, K. Surface morphology and transport properties of $\mathrm{Cr}$ nanoparticles in single electron tunneling regime. J. Phys. Conf. Ser. 266, 012093 (2011).

11. Matsumoto, R. et al. Spin-dependent tunneling in epitaxial $\mathrm{Fe} / \mathrm{Cr} / \mathrm{MgO} / \mathrm{Fe}$ magnetic tunnel junctions with an ultrathin $\operatorname{Cr}(001)$ spacer layer. Phys. Rev. B 79, 174436 (2009).

12. Gaudry, E. et al. From the green color of eskolaite to the red color of ruby: an X-ray absorption spectroscopy study. Phys. Chem. Miner. 32, 710-720 (2006).

13. Goering, E. et al. Strong anisotropy of projected $3 \mathrm{~d}$ moments in epitaxial $\mathrm{CrO}_{2}$ films. Phys. Rev. Lett. 88, 207203 (2002).

14. Vollath, D., Szabó, D. V. \& Willis, J. O. Magnetic properties of nanocrystalline $\mathrm{Cr}_{2} \mathrm{O}_{3}$ synthesized in a microwave plasma. Mater. Lett. 29, 271-279 (1996).

15. He, X. et al. Robust isothermal electric control of exchange bias at room temperature. Nat. Mater. 8, 579-585 (2011).

16. Maekawa, S., Takahashi, S. \& Imamura, H. in Spin Dependent Transport in Magnetic Nanostructures. (eds Maekawa, S. \& Shinjo, T.) Ch. 4 (CRC Press, 2002).

17. Brown, P. J., Forsyth, J. B., Lelièvre-Berna, E. \& Tasset, F. Determination of the magnetization distribution in $\mathrm{Cr}_{2} \mathrm{O}_{3}$ using spherical neutron polarimetry. J. Phys. Condens. Matter. 14, 1957-1966 (2002). 
18. Wiekhorst, F., Shevchenko, E., Weller, H. \& Kötzler, J. Anisotropic superparamagnetism of monodispersive cobalt-platinum nanocrystals. Phys. Rev. B 67, 224416 (2003).

19. Sankar, S., Dieny, B. \& Berkowitz, A. E. Spin-polarized tunneling in discontinuous $\mathrm{CoFe} / \mathrm{HfO}_{2}$ multilayers. J. Appl. Phys. 81, 5512-5514 (1997).

20. Turov, A. E. Can the magnetoelectric effect coexist with weak piezomagnetism and ferromagnetism? Phys. Usp. 37, 303-310 (1994).

21. Bourret, A. et al. Strain relaxation in (0001) AlN/GaN heterostructures. Phys. Rev. B 63, 245307 (2001).

22. Li, Q., Pattada, B., Brueck, S. R. J., Hersee, S. \& Han, S. M. Morphological evolution and strain relaxation of $\mathrm{Ge}$ islands grown on chemically oxidized Si(100) by molecular-beam epitaxy. J. Appl. Phys. 98, 073504 (2005).

23. Bajpai, A. et al. Unusual field dependence of remanent magnetization in granular $\mathrm{CrO}_{2}$ : the possible relevance of piezomagnetism. J. Phys. Cond. Matter. 22, 096005 (2010).

24. Sahoo, S. \& Binek, C. Piezomagnetism in epitaxial $\mathrm{Cr}_{2} \mathrm{O}_{3}$ thin films and spintronic applications. Phil. Mag. Lett. 87, 259-268 (2007).

25. Yang, H., Yang, S. H. \& Parkin, S. S. P. Crossover from Kondo-assisted suppression to co-tunneling enhancement of tunneling magnetoresistance via ferromagnetic nanodots in MgO tunnel barriers. Nano Lett. 8, 340-344 (2008).

26. Kouwenhoven, L. \& Glazman, L. Revival of the Kondo effect. Phys. World 14, 33-38 (2001).

27. Zhuravlev, M. Y., Maekawa, S. \& Tsymbal, E. Y. Effect of spin-dependent screening on tunneling electroresistance and tunneling magnetoresistance in multiferroic tunnel junctions. Phys. Rev. B 81, 104419 (2010).

28. Garcia, V. et al. Ferroelectric control of spin polarization. Science 327, 1106-1110 (2010).

29. Cross, L. E. Relaxor ferroelectrics. Ferroelectrics 76, 241-267 (1987).

30. Rüdiger, A. et al. Nanosize ferroelectric oxides - tracking down the superparaelectric limit. Appl. Phys. A 80, 1247-1255 (2005).

31. Polking, M. J. et al. Ferroelectric order in individual nanometre-scale crystals. Nat. Mater. 11, 700-709 (2012).

32. Shvartsman, V. V., Kleemann, W., Haumont, R. \& Kreisel, J. Large bulk polarization and regular domain structure in ceramic $\mathrm{BiFeO}_{3}$. Appl. Phys. Lett. 90, 172115 (2007).

33. Kimura, T. et al. Magnetic control of ferroelectric polarization. Nature 426, 55-58 (2003).

34. Cheong, S. W. \& Mostovoy, M. Multiferroics: a magnetic twist for ferroelectricity. Nat. Mater. 6, 13-20 (2007).

35. Mostovoy, M., Scaramucci, A., Spaldin, N. A. \& Delaney, K. T. Microscopic theory of temperature-dependant magnetoelectric effect in $\mathrm{Cr}_{2} \mathrm{O}_{3}$. Phys. Rev. Lett. 105, 087202 (2010).

36. Glinchuk, M. D., Eliseev, E. A. \& Morozovska, A. N. Superparaelectric phase in the ensemble of non interacting ferroelectric nanoparticles. Phys. Rev. B 78, 134107 (2008)

37. Pertsev, N. A., Zembilgotov, A. G. \& Tagantsev, A. K. Effect of mechanical boundary conditions on phase diagrams of epitaxial ferroelectric thin films. Phys. Rev. Lett. 80, 1988-1991 (1998).

38. Zavaliche, F. et al. Electric field-induced magnetization switching in epitaxial columnar nanostructures. Nano Lett. 5, 1793-1796 (2005).
39. Khalid, M. et al. Ubiquity of ferromagnetic signals in common diamagnetic oxide crystals. Phys. Rev. B 81, 214414 (2010).

40. Kucheyev, S. O. et al. Electronic structure of chromia aerogels from soft x-ray absorption spectroscopy. J. Appl. Phys. 101, 124315 (2007).

41. Watari, F. \& Cowley, J. M. The study of oxide formation on (001), (011), (111) and (113) surfaces of $\mathrm{Cr}$ thin films using STEM-microdiffraction methods. Surf. Sci. 105, 240-264 (1981)

42. Stierle, A., Koll, T. \& Zabel, H. Structure and defects of epitaxial $\mathrm{Cr}_{2} \mathrm{O}_{3}(0001)$ overlayers on Cr(110). Phys. Rev. B 58, 5062-5069 (1998).

43. Wang, Y. et al. First-principles lattice dynamics, thermodynamics, and elasticity of $\mathrm{Cr}_{2} \mathrm{O}_{3}$. Surf. Sci. 606, 1422-1425 (2012; and references therein

44. Nychka, J. A. \& Clarke, D. R. Damage quantification in TBCs by photostimulated luminescence spectroscopy. Surf. Coat. Tech. 146, 110-116 (2001)

45. Selcuk, A. \& Atkinson, A. Analysis of the $\mathrm{Cr}^{3}+$ luminescence spectra from thermally grown oxide in thermal barrier coatings. Mater. Sci. Eng. A 335, 147-156 (2002).

46. Mougin, J., Le Bihan, T. \& Lucazeau, G. High-pressure study of $\mathrm{Cr}_{2} \mathrm{O}_{3}$ obtained by high-temperature oxidation by $\mathrm{X}$-ray diffraction and Raman spectroscopy. J. Phys. Chem. Sol. 62, 553-563 (2001).

47. McDonaght, C. M. \& Henderson, B. Exchange interactions between $\mathrm{Cr}^{3}+$ ions in magnesium oxide: III. Luminescence and site-selective spectroscopy. J. Phys. C Solid State Phys. 18, 6419-6426 (1985).

\section{Acknowledgements}

We gratefully acknowledge A. Carvalho for SEM observations, J. Arabski for technical assistance during MBE growth, STnano staff for technical support during microfabrication, B. Muller for technical support while setting up DEIMOS beamline, DEIMOS beamline staff for help during synchrotron experiments, and M. Bailleul and V. Da Costa for magnetic and structural characterization measurements. N.N. thanks Région Alsace for financial support.

\section{Author contributions}

D.H. grew the multilayer samples. N.N. fabricated the magnetic tunnel junctions under the supervision of H.M. Y.H. performed the magnetotransport measurements. L.J., P.O. and F.S. carried out the synchrotron experiments. C.U.-B. made the TEM observations. S.B. measured the micro-luminescence spectra. D.H. and Y.H. analysed the results. D.H, B.D. and Y.H. prepared the manuscript.

\section{Additional information}

Supplementary Information accompanies this paper at http://www.nature.com/ naturecommunications

Competing financial interests: The authors declare no competing financial interests.

Reprints and permission information is available online at http://npg.nature.com/ reprintsandpermissions/

How to cite this article: Halley, D. et al. Size-induced enhanced magnetoelectric effect and multiferroicity in chromium oxide nanoclusters. Nat. Commun. 5:3167 doi: $10.1038 /$ ncomms4167 (2014). 